\title{
Resultados de la implantación del "Programa de cuidado de la salud física para pacientes con trastorno mental grave"
}

Findings of the Implementation of a Program to Monitor the Physical Health of Patients with Severe Mental Disorders

\author{
Patricia Lana Elola \\ Unidad de Salud Mental Comunitaria Santa Cruz/Salamanca, Santa Cruz de Tenerife, España. \\ Contacto: patrilana@hotmail.com \\ Fecha de recepción: 1 de julio de 2016 / Fecha de aceptación: 18 de octubre de 2016
}

\begin{abstract}
Resumen
La preocupación por la salud física del paciente con trastorno mental grave (TMG) es creciente dada la mayor incidencia de comorbilidad de patologías físicas y la reducción en su esperanza de vida. Desde 2010 se instauró en la Unidad de Salud Mental (USM) Santa Cruz-Salamanca un "Programa de cuidado de la salud física para pacientes con TMG" con el objetivo de evaluar el estado de salud de nuestros pacientes, detectar los casos de riesgo cardiovascular (RCV) elevado, la presencia en ellos de síndrome metabólico (SM) e iniciar un primer paso de intervención individualizada. Añadimos en las consultas de enfermería una valoración analítica completa, registro de constantes, medidas antropométricas y una entrevista sobre hábitos dietéticos y de estilo de vida; correlacionamos estos datos e iniciamos un primer nivel de educación sanitaria, derivando además a Atención Primaria los casos de riesgo para la salud. Se han ido incluyendo en el mismo a todos los pacientes que son atendidos en la Consulta de Enfermería de la USM y a pacientes con TMG derivados por otros profesionales de la unidad. En el momento de la inclusión de los pacientes en el programa, un $42,7 \%$ presentaban obesidad (grados I, II, III o IV), el riesgo cardiovascular medio era de un $7,5 \%$ en la escala de Anderson y un $34 \%$ presentaban síndrome metabólico. Tras 4 años de desarrollo del programa, el $47,6 \%$ de los pacientes han bajado de peso, se ha mantenido la media de RCV y se redujo a un $32,8 \%$ los pacientes que siguen presentando SM.
\end{abstract}

Palabras clave: salud mental, trastorno mental grave (TMG), riesgo cardiovascular (RCV), síndrome metabólico (SM), unidad de salud mental comunitaria (USMC).

\begin{abstract}
The concern for the physical health of patients with severe mental disorder is increasing due to a higher incidence of co-morbility and reduced life expectancy in those patients. In 2010, a programme to monitor the physical health of patients with severe mental disorders was set up in Unidad de Salud Mental Santa Cruz-Salamanca aimed to monitor the cardiovascular risk and detect cases of metabolic syndrome, as well as to initiate a personalised intervention. A complete set of analysis, anthropometric measurements and an interview about life-style took place when the patients had appointment with the nurse. Those results were then used to raise awareness about health education with the patients and to refer some cases to the general practitioners. All patients attended by the mental health nurse took part in this programme, plus some other patients sent from other professionals of the unit.

In the beginning of the programme $42.7 \%$ of the patients were obese (I, II, III and IV degrees), cardiovascular risk according to the Anderson scale was 7.5 and $34 \%$ had metabolic syndrome. After 4 years, $47.6 \%$ of patients had lost weight, the average cardiovascular risk was unchanged despite the age and patients presenting metabolic syndrome reduced to $32.8 \%$.
\end{abstract}

Keywords: mental health, severe mental disorders, cardiovascular risk, metabolic syndrome, community mental health. 


\section{Introducción}

El modelo actual de atención comunitaria a los problemas de salud mental es el resultado de la reestructuración de los recursos asistenciales propuesta en los años 80 en el Informe de la Comisión Ministerial para la Reforma Psiquiátrica (MSC, 1985) ${ }^{1}$ y plasmada en la Ley General de la Sanidad (LGS, 1986) ${ }^{2}$. Las unidades de salud mental comunitaria (USMC) son el eje sobre el que se estructura el modelo comunitario de atención a la salud mental; están constituidas por equipos multidisciplinares que ofrecen una atención integral de las necesidades psicológicas, biológicas y del entorno sociofamiliar de los usuarios. Además de la función asistencial se realiza promoción de la salud y prevención de la enfermedad desde un ámbito cercano al lugar de residencia de la población de referencia. Dentro de la atención a la salud mental de la población derivada a nuestra USM, debemos emplear gran parte de nuestro tiempo y esfuerzo en la atención del paciente diagnosticado de un trastorno mental grave (TMG), dada la severidad y cronicidad de los mismos. La definición de TMG que cuenta con mayor consenso es la que emitió el Instituto Nacional de Salud Mental de EE. UU. en 1987 (NIMH, 1987) ${ }^{3}$, y que incluye tres dimensiones:

1. Diagnóstico: Incluye a los trastornos psicóticos (excluyendo los orgánicos) y algunos trastornos de la personalidad.

2. Duración de la enfermedad y del tratamiento: Tiempo superior a los dos años.

3. Presencia de discapacidad: Existencia de una disfunción moderada o severa del funcionamiento global, medido a través del GAF (Global Assessment of Functioning APA, 1987), que indica una afectación de moderada a severa del funcionamiento laboral, social y familiar.

El criterio diagnóstico incluye principalmente a las personas que padecen algún tipo de psicosis funcional (no orgánica), que en la clasificación OMS CIE-10 incluye los códigos F20-F22, F24, F25, F28-F31, F32.3, F33.3 (Gisbert, 2003) ) $^{4,5}$.

Desde 2010 y a sugerencia de la Dirección General de Programas Asistenciales del Servicio Canario de la Salud se implantó un "Programa de cuidado de la salud física en el paciente con trastorno mental grave" ${ }^{, 6,7}$ para cubrir una carencia detectada, ya que el paciente con TMG es atendido en el circuito psiquiátrico, pero con frecuencia no asiste regularmente a su médico de atención primaria ni a los programas de prevención previs- tos desde ese nivel asistencial. Los objetivos en la implantación del programa fueron:

1. Evaluar el estado de salud física de los pacientes en el inicio en el programa.

2. Detectar la presencia de RCV elevado y/o síndrome metabólico en la población.

3. Implementar un programa de intervención personalizado del cuidado de la salud física de cada paciente.

4. Derivar a atención primaria los casos de elevado riesgo $\mathrm{CV}$ o SM.

5. Analizar la evolución en el tiempo de los parámetros de salud física tras la inclusión del paciente en el programa.

Todo el equipo se involucró en su implementación, si bien la enfermera juega un papel preponderante en ello. En la actualidad hay consenso y una creciente preocupación sobre la salud física del paciente psiquiátrico grave; entre el 50 y el $70 \%$ de los pacientes con TMG presentan comorbilidades físicas (Jones y Macías, 2004 ${ }^{8}$; Joffre y Maldonado, 20099; Bruisan, $2012^{10}$ ), siendo las más frecuentes las de tipo cardiovascular y metabólicas. La tasa de muerte por enfermedad cardiovascular del paciente con TMG es más de un $50 \%$ superior al resto de la población (Hennekes\&Hennekes, 2005) ${ }^{11}$ y su esperanza de vida se ve reducida en un 20\% (Bobes, 2010) ${ }^{12}$. Además los pacientes con TMG diagnosticados de síndrome metabólico duplican a la población general (Bobes, 2010). Todo esto, junto con características propias de la enfermedad mental grave (disminución del impulso a la actividad, abulia, clinofilia, retraimiento social, gran incidencia de tabaquismo, estigma...) incrementa la necesidad de actuación para detectar y tratar posibles complicaciones.

Por otra parte, la cercanía de las USM al paciente y la frecuencia de la consultas de enfermería especializada (cada semana, 2, 3 o 4 semanas) (Lana, 2009) $)^{13}$, hacen que el paciente tenga una gran adherencia a su unidad y una estrecha relación enfermera/paciente que facilita la puesta en marcha de planes de detección y cuidado. Además hay factores de riesgo que son modificables desde nuestras consultas, como son el tabaquismo, el sedentarismo, los hábitos dietéticos y la medicación administrada. Los últimos tratamientos antipsicóticos, tanto orales como intramusculares de liberación prolongada, buscan un buen perfil de eficacia pero mejorando la tolerabilidad con menores efectos secundarios a nivel neurológico y también respecto 
al incremento ponderal y el riesgo cardiovascular. Por tanto, se hace imprescindible la coordinación entre atención primaria y especializada para mejorar el estado de salud de este colectivo.

\section{Objetivos}

1. Describir el estado de salud física de la muestra en términos de RCV y síndrome metabólico

2. Describir la evolución de los principales parámetros de salud física a lo largo de cuatro años de desarrollo del programa.

\section{Material y método}

Se trata de un estudio longitudinal prospectivo, que se realiza en la Unidad de Salud Mental Comunitaria (USMC) Santa Cruz-Salamanca, perteneciente al Área Sur del Servicio Canario de Salud de Santa Cruz de Tenerife. Esta USMC atiende a una población de referencia de 124147 habitantes.

\section{Duración del estudio}

Hacemos un corte en el periodo comprendido entre enero del 2010 y marzo del 2014. El programa continúa abierto en la actualidad.

\section{Criterios de inclusión/exclusión}

Inclusión:

1.Pacientes con TMG en seguimiento en la USMC de Santa Cruz-Salamanca.

Exclusión:

1. Abandono del seguimiento en consultas.

2. Residencia del paciente en algún dispositivo del Equipo Comunitario Asertivo (ECA) de la zona.

3. Para el análisis evolutivo (apartado 4.2.) se excluyen los pacientes incluidos en el programa con posterioridad a 2011.

\section{Tamaño, características y distribución de la muestra}

Total: 152 pacientes que cumplían los criterios de inclusión. Son captados por acudir regularmente a consultas de enfermería para administración del tratamiento y/o control analítico o derivados por el psiquiatra por presentar factores de riesgo. De los cuales:

- 68 acuden regularmente (administración de medicación depot).

- 35 acuden para control analítico (clozapina).
- 28 acuden para control analítico + inyectables depot.

- 18 acuden derivados por su psiquiatra (susceptibles de riesgo $\mathrm{CV}$ ).

Edad media: 46 años.

Distribución por género:

$69 \%$ hombres.

$31 \%$ mujeres.

Recursos humanos

1. 1 enfermera especialista en salud mental de la USMC.

2. Personal de laboratorio del Instituto de Atención Socio-Sanitaria(IASS).

Recursos Materiales

1. Consultorio:

- Pesa con tallímetro.

- Cinta métrica.

- Tensiómetro.

- Fonendoscopio.

- Material de punción y tubos para recogida de muestras.

- PC e impresora. Software estadístico básico.

- Tablas para la medición del RCV (Anderson).

- Criterios diagnósticos del síndrome metabólico de la NCEP-ATPIII.

Variables de estudio

1. Sexo.

2. Edad.

3. TA.

4. Peso.

5. Talla.

6. IMC.

7. Perímetro abdominal.

8. Colesterol total.

9. HDL Colesterol.

10. LDL Colesterol.

11. Triglicéridos.

12. Glucemia.

13. Tabaco (n. ${ }^{\circ}$ cigarrillos/día).

14. Entrevista (hábitos dietéticos, estilo de vida y actividad física diaria).

La mayoría de los pacientes con TMG acuden a la consulta de enfermería en USM cada 2, 3 o 4 semanas; en una de esas visitas, además de las intervenciones propias, pactamos con carácter anual la revisión del 
Programa de SF. Se realiza una extracción de análitica sanguínea (hemograma, ionograma, perfil lipémico, glucemia, perfil renal, hepático y hormonas tiroideas), toma de constantes (T/A, FC), medidas antropométricas (peso, talla, IMC y perímetro abdominal) y realización de una entrevista sobre hábitos dietéticos y estilo de vida (dieta que lleva el paciente, conocimientos y motivos, consumo de tabaco y/o tóxicos, actividades de la vida diaria, horas de sueño/actividad, motivos...).

Posteriormente se correlacionan los resultados analíticos con el resto de las variables utilizando la Tabla de Cálculo del Riesgo Coronario de Anderson (1991) y la tabla de conversión de los mismos en riesgo coronario (fig. 1).

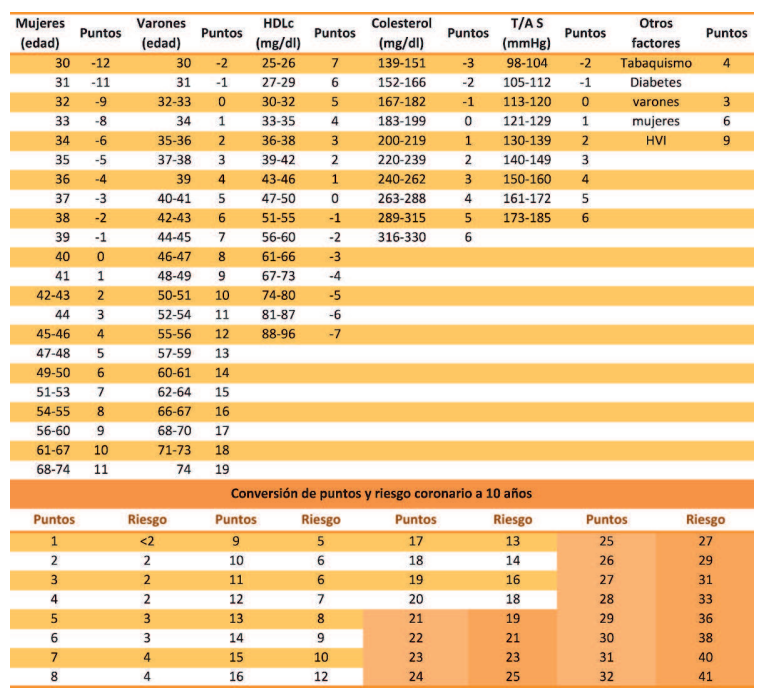

Figura 1: Tabla de RCV de Anderson

El índice de RCV nos muestra en un porcentaje la probabilidad de que una persona sufra una enfermedad cardiovascular (cardiopatía isquémica, accidente cerebrovascular, insuficiencia cardiaca isquémica y/o hipertensiva, arteriopatía periférica) en los próximos 10 años. Se usa esta herramienta por su sencillez y amplia difusión que permite el cotejo de datos.

Las variables recogidas también correlacionan en la Tabla de Criterios Diagnósticos del Síndrome Metabólico de la NCEP-ATPIII (fig. 2), para hallar el RCV de cada paciente y la presencia o no de síndrome metabólico.

\begin{tabular}{|l|r|}
\hline Factor de riesgo & Definición \\
\hline Obesidad abdominal & $\begin{array}{r}\text { Perímetro abd. }>102 \mathrm{~cm} \text { en hombres } \\
88 \mathrm{~cm} \text { en mujeres }\end{array}$ \\
\hline Triglicéridos altos & $>150 \mathrm{mg} / \mathrm{dl}$ \\
\hline Colesterol HDL bajo & $<40 \mathrm{mg} / \mathrm{dl}$ en hombres \\
& $<50 \mathrm{mg} / \mathrm{dl}$ en mujeres \\
\hline Hipertensión arterial & $>130 / 85 \mathrm{~mm} \mathrm{Hg}$ \\
\hline Hiperglucemia en ayunas & $>110 \mathrm{mg} / \mathrm{dl}$ \\
\hline
\end{tabular}

Figura 2: Criterios diagnósticos de SM NCEP-ATPIII

El síndrome metabólico ( $\mathrm{SM}$ ) es la conjunción en un individuo de múltiples factores de riesgo, cuyo nexo común es el aumento de la resistencia a la insulina y el exceso de grasa abdominal que le otorgan un riesgo elevado de desarrollar una enfermedad cardiovascular. Se hace el diagnóstico de SM cuando están presentes 3 o más de los factores de riesgo descritos.

Los datos se almacenan en hojas de calculo (EXCEL) y se hallan con dicho programa los indicadores de moda, media aritmética, promedio, etc. Los datos se tratan con el programa de análisis estadístico G-Stat versión 2.0 y el test no paramétrico de Wilcoxon para las variables cuantitativas.

En caso de presentar un RCV moderado o elevado (> $16 \%$ ) y/o presencia de 3 o más criterios diagnósticos de SM, se le remite a su MAP con una carta de derivación para su control (fig. 3).

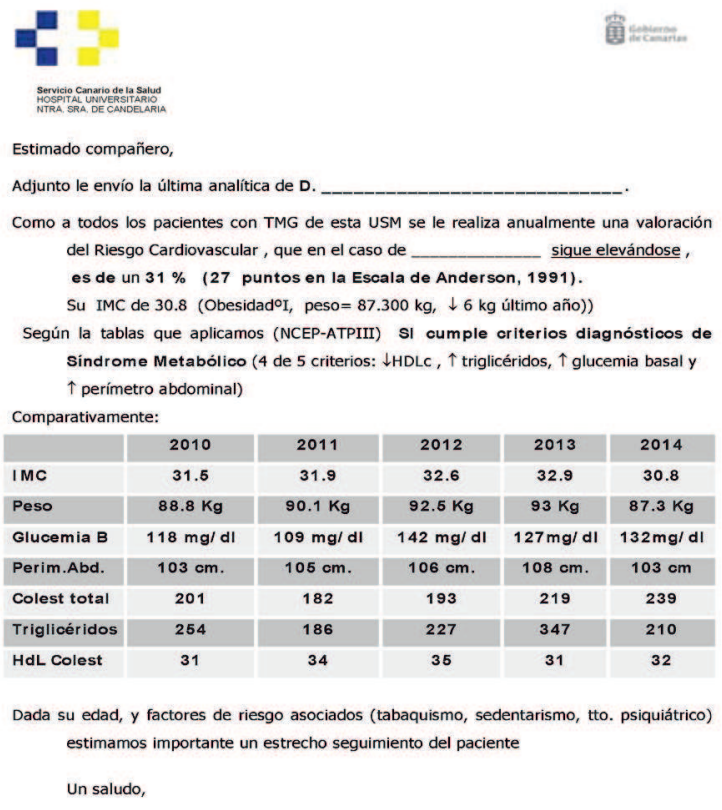

Figura 3: Ejemplo de carta de derivación a MAP 


\section{Resultados}

\subsection{Estado de salud del paciente con TMG en el momento de su inclusión en el programa}

En esta sección expondremos una visión general del estado de salud de los 152 pacientes en su primer contacto con el Programa de Salud Física. Tan sólo un $21,7 \%$ presenta un IMC normal; un 34,21\%, sobrepeso y un $42,7 \%$ de la muestra presenta obesidad en grados I, II o III (fig. 4). La media es de 29,6 (sobrepeso).

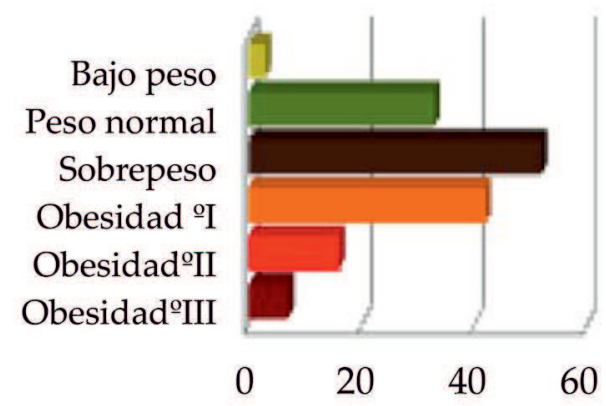

Figura 4. Distribución del IMC de la muestra.

El 80,2\% de los pacientes presentan cifras de T/A sistólica inferior a $130 \mathrm{mmHg}$ y en el 56,6\% la T/A diastólica es inferior a $80 \mathrm{mmHg}$. Un $15 \%$ son diabéticos.

Respecto al hábito tabáquico, el $44 \%$ de los pacientes no fuma, el $56 \%$ es fumador y la media de 29 cigarrillos consumidos al día (fig. 5).

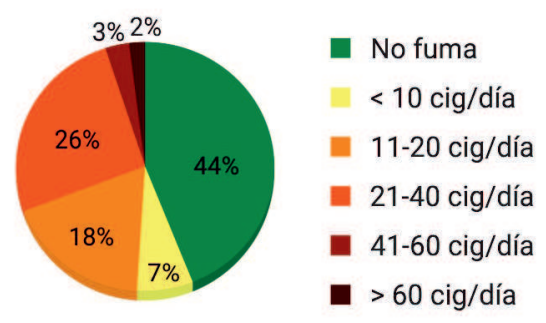

Figura 5: Número de cigarrillos/día

En la entrevista personal el $73 \%$ se considera activo, que hace ejercicio de forma regular o permanece mayor tiempo en movimiento que sentado o tumbado.

Respecto a los resultados analíticos, cabe destacar que el $72,36 \%$ mantiene unas cifras de colesterol total y triglicéridos normales, inferiores a $220 \mathrm{mg} / \mathrm{dl}$ y 200 $\mathrm{mg} / \mathrm{dl}$ respectivamente. El HDL colesterol es normal (> a $35 \mathrm{mg} / \mathrm{dl}$ ) en el 74,43\% de la muestra examinada (fig. 6).
Dentro LN 75\%

Fuera LN 25\%

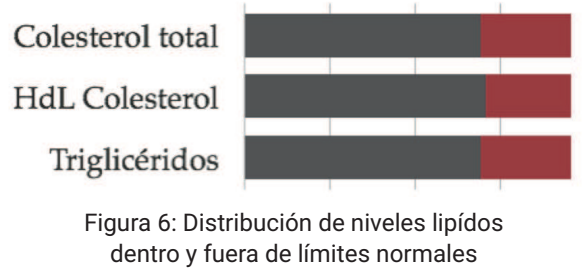

Todos estos datos analizados en su conjunto nos dejan una media de riesgo cardiovascular de un 7,5\%. Tan sólo un 6,6\% de los pacientes presenta un RCV > a $16 \%$, cifra a partir de la cual se considera un riesgo elevado y es necesaria su derivación para seguimiento especializado (fig. 7).

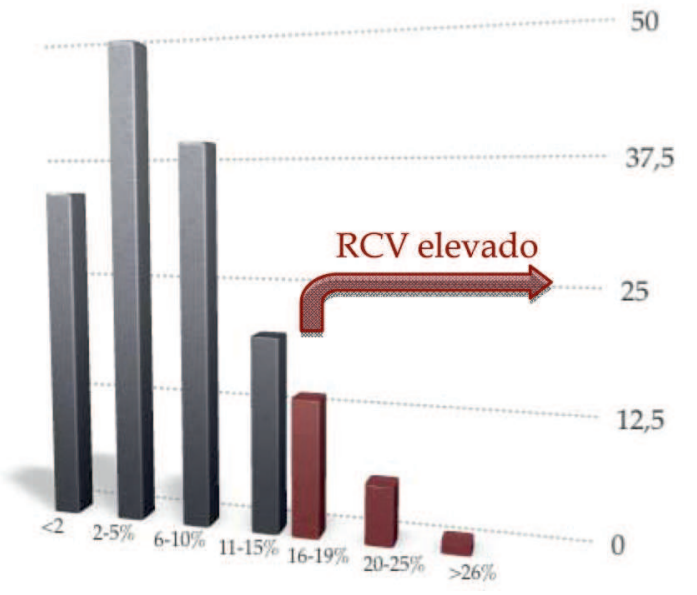

Figura 7: Distribución del RC

Respecto a la existencia de síndrome metabólico, el $66 \%$ de los pacientes cumple 2 o menos criterios diagnósticos y el $34 \%$, sin embargo, puede ser diagnosticado de SM por cumplir 3 o más (fig. 8).

\section{0}

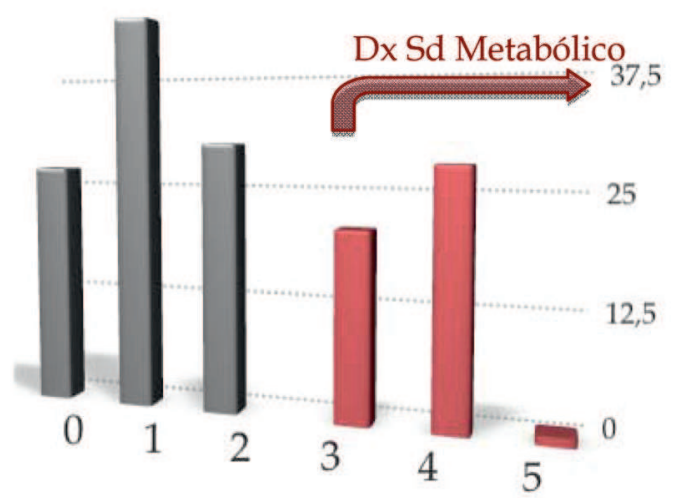

Figura 8: Distribución de los criterios dx SM 
Con todos estos datos, tras una primera valoración por Enfermería, se derivó al centro de salud al $35 \%$ de los pacientes para dar a conocer a su MAP esta situación y recibir tratamiento o seguimiento según los protocolos de atención primaria.

\subsection{Análisis de la evolución de los pacientes en seguimiento en el programa}

Para el análisis de los datos de esta parte se ha seleccionado una muestra de 84 pacientes incluidos en el Programa de Salud Física de la USM S/C-Salamanca. La muestra es inferior a la del capítulo anterior porque para ver el evolutivo seleccionamos a aquellos de los cuales tengamos registros anuales de 2010 a marzo de 2013, quedando excluidos pacientes introducidos en el programa con posterioridad a 2011.

Respecto al peso, un 47,6\% bajaron de peso, un $17,8 \%$ se mantuvieron y un $34,6 \%$ de los pacientes subió de peso, y de forma paralela se comportaron los parámetros de IMC y perímetro abdominal (fig. 9).

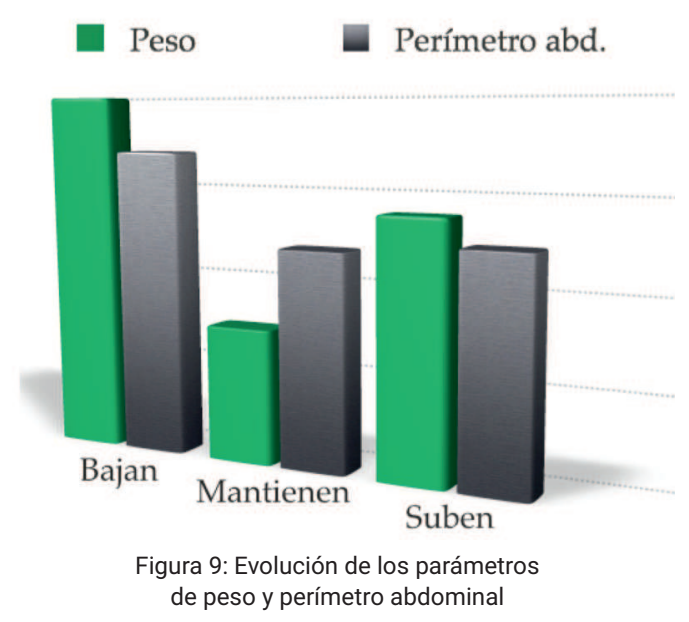

E1 90,4\% se mantuvieron normotensos. Se diagnosticó un nuevo caso de diabetes mellitus tipo II. Se produjeron 5 fallecimientos (2 IAM, 1 septicemia y 2 oncológicos).

Un sólo paciente dejó de fumar en estos años y el $68 \%$ de los pacientes mantuvieron un consumo similar en número de cigarrillos.

Un $16 \%$ de los pacientes que previamente se definían como sedentarios incrementó su actividad física diaria y tan sólo 1 de ellos la disminuyó.
En los controles sucesivos se objetivó un descenso no estadísticamente significativo de las cifras de colesterol, HDL colesterol y triglicéridos (fig.10).

\section{Colesterol HdL Colesterol Triglicéridos}

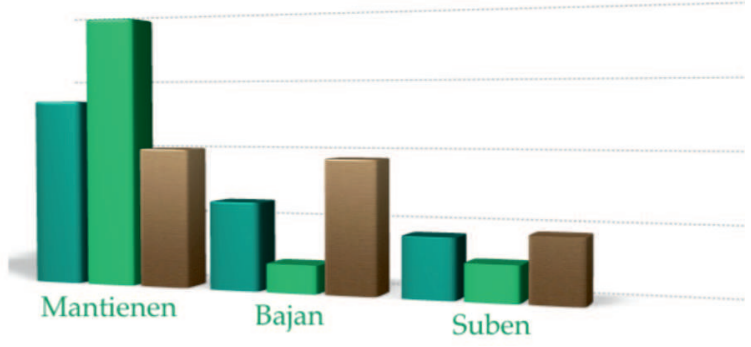

Figura 10: Relación de la evolución de los valores lipémicos

En las sucesivas valoraciones anuales del RCV, el $38 \%$ de los pacientes mantienen el mismo porcentaje de $\mathrm{RCV}$, un $24 \%$ rebaja su riesgo $\mathrm{CV}$ y un $38 \%$ incrementa el porcentaje de RCV.

Respecto al cumplimiento de criterios diagnósticos de SM evolutivamente, el $57 \%$ de los pacientes sigue cumpliendo el mismo número de criterios, un $29 \%$ reduce el n. ${ }^{\circ}$ de criterios que previamente cumplía y un $21 \%$ de la muestra pasa cumplir más a lo largo de los años (fig. 11).

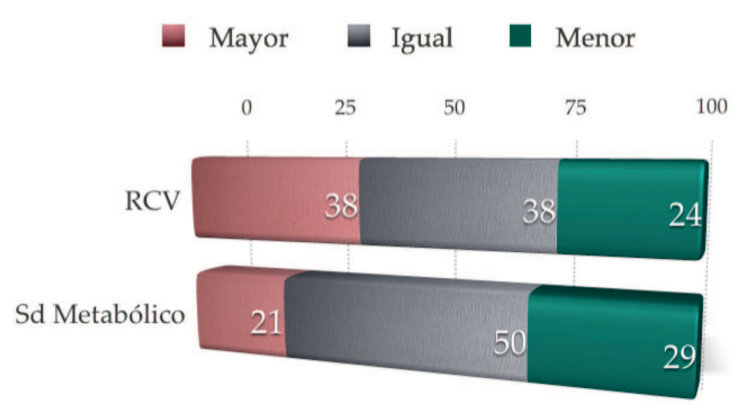

Figura 11: Evolución de los parámetros de RCV y SM (\%)

\section{Discusión y conclusiones}

Cabe destacar la coincidencia de los datos hallados con estudios previos que alertaban sobre la mayor incidencia de SM en pacientes con TMG; el 34\% de los pacientes valorados fueron diagnosticados de SM, así que coincidimos con Sanchez-Araña ${ }^{14}$ que lo diagnosticó en el $36 \%$ de los pacientes ingresados en el Hospi- 
tal Dr. Negrín de Las Palmas de Gran Canaria. La muestra analizada en el presente estudio consta de pacientes crónicos de largo tiempo de evolución y una edad media de 46 años, mientras que el perfil de paciente ingresado en UIB suele ser más joven y de reciente diagnóstico. Si tomamos los datos de Cordero ${ }^{15}$ en los que estimaba que el $24 \%$ de la población general cumple criterios diagnósticos de SM, no coincidimos con Bobes $^{12}$ en la duplicación de las tasas, pero sí concluimos en que los datos de SM en TMG son más elevados que en la población general. Vera, Aguado y col. ${ }^{16}$ lo diagnosticaban entre en $41,46 \%$ en 2007 y un $21,95 \%$ en 2012 en el ámbito de una unidad de rehabilitación, así que coincidimos en que la intervención de enfermería especializada en salud mental contribuye a reducir su prevalencia.

También las cifras de SM de nuestro estudio resultan más elevadas que las de población canaria analizada por Cabrera de León y col. ${ }^{17}$, que alcanzan a un $24 \%$ de una muestra de 6729 personas con una edad media de 43 años, que resultaban a su vez similares a los datos de la $\operatorname{ENCA}(24,4 \%)^{18}$ y los de la población estadounidense $(21,8 \%)^{19}$. Por tanto, concluimos que la presencia de SM es superior entre la población con $\mathrm{TMG}$.

Los datos encontrados de sobrepeso y obesidad, sin embargo, coinciden con los de la muestra de Cabrera y León ${ }^{16}$ (población canaria, no específicamente psiquiátricos) de un $70 \%$ de la población estudiada. Diferimos con los datos de la Encuesta Nacional de Salud $(\text { ENS })^{20}$, que arroja datos de un 37,4\% de los adultos en sobrepeso y un $15,2 \%$ en obesidad, ya que nuestra muestra es muy superior, de un 34,2\% y $42,7 \%$ respectivamente.

El índice de diabéticos encontrados (15\%) es cercano al de la muestra de Cabrera y León ${ }^{16}$ (12,5\% una vez estandarizado el sexo y la edad) en población canaria.

Otro dato relevante es el índice de tabaquismo ( $56 \%$ de fumadores) de la muestra, que, aunque elevado, es menor de lo que pensábamos a priori encontrarnos. Sin embargo, son mayores que los que arroja el Informe Vital Signs ${ }^{21}$ sobre tabaquismo en TMG en EE. UU. (40\% de los varones y $34 \%$ de las mujeres) y del encontrado por Cabrera y León ${ }^{16}$ en la población canaria.

No encontramos publicaciones respecto a las cifras de $\%$ de RCV en pacientes con TMG. En nuestra muestra interpretamos positivamente el dato de que un $62 \%$ mantenga o disminuya su porcentaje de RCV a la largo de los 4 años analizados, ya que la edad en sí misma incrementa la puntuación en las tablas empleadas.

La baja tasa de sedentarismo (23\%) puede deberse a la metodología empleada (valoración subjetiva del paciente y el profesional analizando las actividades de la vida diaria) y podría ser medida con alguna escala validada en estudios posteriores.

Respecto a los resultados en el estudio comparativo, cabe destacar la mejoría a lo largo de los años en todos los parámetros estudiados. En la evolución del peso, perímetro abdominal e IMC, nos parece destacable que casi la mitad de la población consiga bajar de peso a lo largo de los años. Incluso se hallaron diferencias estadísticamente significativas en submuestras en las se analizaron las variaciones en relación con los cambios en el antipsicótico principal ${ }^{22}$, dato que nos ha incentivado a seguir investigando en esta línea.

También hallamos mejorías no estadísticamente significativas en los valores analíticos de colesterol total y triglicéridos, y en mucho menor grado de HDL colesterol, que se mantiene casi invariable.

Como punto de mejora pensamos en el establecimiento de un feedback con Atención Primaria, ahora que compartimos soportes informáticos, para cuantificar si los pacientes derivados realmente están llegando o mejorar la comunicación en esos casos.

Cabe destacar la buena aceptación que ha tenido la implementación del Programa de Salud Física entre los pacientes con TMG de la USM, ya que es un indicador objetivable del estilo de vida del paciente, de su estado de salud, y nos sirve como elemento de refuerzo positivo en nuestro desempeño diario. Hablando de aspectos físicos y estilos de vida, también tratamos aspectos de la sintomatología propia del paciente con TMG y amplía el tratamiento holístico del mismo. Tanto en los casos que se derivan a Atención Primaria como en los que no, se ha iniciado un primer nivel de educación sanitaria sobre hábitos de alimentación y estilo de vida sana que enriquecen los cuidados que recibe el paciente con trastorno mental grave en la unidad y son claves en la mejora de los parámetros analizados. La frecuencia de las visitas del paciente con TMG a consulta de enfermería facilita esa labor de educación y seguimiento, si bien consideramos una fortaleza la implicación del resto del equipo para el éxito del programa, sobre todo los psiquiatras en su papel de motivación y 
revisión de los tratamientos administrados, buscando estabilidad psicopatológica y disminución de la sintomatología negativa que predisponga al paciente a los cambios.

Por tanto, continuamos año tras año con el programa y animamos a su implantación en otras unidades de salud mental, así como al registro y publicación de experiencias similares.

\section{Bibliografía}

1. Ministerio de Sanidad y Consumo. Informe de la Comisión Ministerial para la Reforma Psiquiátrica, Secretaría General Técnica. Madrid : Ministerio de Sanidad y Consumo, 1985

2. Ley General de Sanidad. Ley $14 / 1986$ de 25 de Abril. BOE n 102 del 29/4/1986. Ministerio de Sanidad y Consumo. Madrid, 1986

3. National Institute of Mental Health. Towards a model for a comprehensive community based mental health system. Washington DC: NIMH; 1987.

4. Gisbert C, Arias P, Camps C, Cifre A, Chicharro F, Fernández J, et al. Rehabilitación psicosocial del trastorno mental severo: situación actual y recomendaciones. Madrid: Asociación Española de Neuropsiquiatría; 2002. Cuadernos Técnicos, n. 6.

5. Gisbert Aguilar, C. Rehabilitación psicosocial y tratamiento integral del TMG Ponencia. Congreso Asoc Nac Española de Neuropsiquiatría, Oviedo, Junio 2003

6. http://www3.gobiernodecanarias.org/sanidad/scs. Consultado en Mayo 2016

7. Guía “La salud física del paciente con trastorno mental grave” Consejería de Sanidad, Gobierno de Canarias

http://www3.gobiernodecanarias.org/sanidad/scs/content/636 f108f-f896-11df-8010

c5c56db520fc/Gu\%C3\%ADa\%20de\%20cuidados\%20fisicos\%20 definitiva\%20completa.pdf. Consultado en Mayo 2016

8. Jones DR, Macias C, Barreira PJ, Fisher WH, Hargreaves WA, Harding CM. Prevalence, severity, and co-occurrence of chronic physical health problems of persons with serious mental illness. Psychiatr Serv. 2004 Nov;55(11):1250-7.

9. Joffre-Velazquez et al. Enfermedad psiquiátrica y síndrome metabólico. Énfasis en el trastorno esquizofrénico. Revista Médica del Hospital General de México, vol 72, nº 1 Enero-Marzo, pp41-49

10. Bruisan, Enric .Deterioro cognitivo y síndrome metabólico en esquizofrenia.

http://www.forumclinic.org/es/esquizofrenia/noticias/deteriorocognitivo-y-s\%C3\%ADndrome-metabólico-en-la-esquizofrenia. Consultado en Mayo 2016

11. Hennekens $\mathrm{CH}$, Hennekens AR, Hollar D, Casey DE. Schizophenia and increased risks of cardiovascular disease. Am Heart J 2005; 150: $1115-21$

12. Bobes J, Arango C, Aranda P, Carmena R, Garcia-Garcia M, Rejas J; Cardiovascular and metabolic risk in outpatients with schizophrenia treated with antipsychotics: results of the CLAMORS Study. CLAMORS Study Collaborative Group. Schizophr Res. 2007 Feb;90(1-3):162-73. Epub 2006 Nov 21

13. Lana Elola, P; Enfermería en la Unidad de Salud Mental Santa CruzSalamanca. ENE Revista de Enfermería 3 (3): 35-43, Dic 2009.

14. Sanchez-Araña Moreno T. Esquizofrenia y síndrome metabólico: análisis de la prevalencia (Tesis doctoral), Enero 2008

15. Cordero Alegrís, L. Prevalencia de Sd Metabólico, Revista Española de Cardiología 2005, Vol.5, Supl D.

16. Vera García, Elisa María; Aguado Pacheco, Rocío; Castaño Alba, Miguel Ángel; López González, Sara; et al.. Evolución de la prevalencia de síndrome metabólico en personas con trastorno mental grave e intervención enfermera. Rev Presencia 2014 jul-dic,
10(20). Disponible en:

<http://www.index-f.com/presencia/n20/p9358.php> Consultado el 17 de Diciembre de 2015

17. Cabrera de León, Antonio; Rodríguez Pérez, Ma. del Cristo, Almeida González, Delia y col. Presentación de la cohorte "CDC de Canarias": objetivos, diseño y resultados preliminares. Revista Española de Salud Pública. Versión impresa ISSN 1135-5727. Rev. Esp. Salud Publica v.82 n. 5 Madrid set.-oct. 2008

18. Álvarez León EE, Ribas Barba L, Serra Majem LI. Prevalencia del síndrome metabólico en la población de la Comunidad Canaria. Med Clin (Barc). 2003; 120:172-74.

19. Ford ES, Giles WH, Dietz WH. Prevalence of the metabolic syndrome among US adults. JAMA. 2002; 287: 356-59.

20. Encuesta Nacional de Salud 2006. Resultados pre- liminares (marzo 2007). Ministerio de sanidad y Consumo. Gobierno de España. Disponible en:

http://www.msssi.gob.es/va/estadEstudios/estadisticas/encu estaNacional/encuestaNac2006/encuestaNacionalSalud2006. pdf. Consultado en Mayo 2016

21. Vital Signs: Current Cigarette Smoking Among Adults Aged $\geq 18$ Years with Mental Illness - United States, 2009-2011 WeeklyFebruary 8, 2013 / 62(05);81-87

http://www.cdc.gov/tobacco/campaign/tips/resources/data/ci garette-smoking-in-united-states.html\#mental-health. Consultado en Mayo 2016

22. Gallego González JA, Lana Elola P, Criado Gutierrez I. Evaluación de eficacia clínica, salud física y síndrome metabólico de palmitato de paliperidona en pacientes con TMG ambulatorios previamente estabilizados con RILD. Poster presentado en el XVI World Congress of the World Psychyatry Association, Madrid 2014. 